\title{
Reporte de un caso con reacción a la polisulfona durante hemodiálisis. Clínica Reynadial, Guayaquil, 2020
} Report of a case with a reaction to polysulfone during hemodialysis. Reynadial Clinic, Guayaquil, 2020

\author{
Mario Hernández ${ }^{1}$, Elsa Bernal², Fresia Massuht ${ }^{3}$, Emilio Fors ${ }^{1}$, Yinet Ramírez ${ }^{4}$, Ingrid Figueredo ${ }^{4}$, Raúl Caballero ${ }^{4}$, Martha Fors $^{5}$
}

DOI. 10.21931/RB/2020.05.04.19

Resumen: El tratamiento de hemodiálisis (HD) presupone un cierto riesgo de reacciones adversas de hipersensibilidad, se han descrito casos de hipersensibilidad con membranas biocompatibles, como la polisulfona. En este artículo describimos el caso de un paciente que se realiza tratamiento de HD usando dializador de polisulfona, aproximadamente a los 10 minutos de iniciar presenta manifestaciones digestivas, caracterizada por diarreas liquidas, no relacionadas con evento alimenticio, sin flemas, ni contenido hemático, sin pujos ni tenesmo, presenta dolor abdominal difuso, vómitos e hipotensión arterial. Estas reacciones fueron consideradas como hipersensibilidad relacionada con el proceso de hemodiálisis, el cuadro clínico no reapareció en observaciones sucesivas cuando las sesiones se llevaron a cabo utilizando una membrana de celulosa.

Palabras clave: Hemodiálisis, membrana de polisulfona, reacción de hipersensibilidad.

Abstract: Hemodialysis (HD) treatment assumes a certain risk of adverse hypersensitivity reactions; cases of hypersensitivity with biocompatible membranes, such as polysulfone, have been described. In this article we describe the case of a patient who undergoes HD treatment using polysulfone dialyzer, approximately 10 minutes after starting, he presents digestive manifestations, characterized by liquid diarrhea, not related to eating events, without phlegm, or blood content, without straining or tenesmus, presents diffuse abdominal pain, vomiting and arterial hypotension. These reactions were considered as hypersensitivity related to the hemodialysis process, the clinical picture did not reappear in successive observations when the sessions were carried out using a cellulose membrane.

Key words: Hemodialysis, polysulfone membrane, hypersensitivity reaction.

\section{Introducción}

El realizar una sesión de hemodiálisis (HD) puede conllevar a riesgo de aparición de reacciones adversas de hipersensibilidad para el paciente, al estar su sangre en contacto con diferentes materiales de origen sintético ${ }^{1}$. Se ha descrito por varios autores reacciones de hipersensibilidad al óxido de etileno y a membranas de baja biocompatibilidad como el cuproamonio. También se han reportado casos de hipersensibilidad con membranas biocompatibles como la polisulfona, e incluso con polisulfona asociada a polivinilpirrolidona (PVP)1,2.

En la literatura hay ejemplos de reacciones anafilácticas y anafilactoides o intolerancia a distintos tipos de membranas sintéticas en el mismo paciente ${ }^{3,4,5}$, lo cual se atribuye a las diferencias en manufactura y a la cantidad de PVP utilizada para hidrofilizar la membrana.

Se ha evidenciado ausencia de reacción anafilactoide con el uso de un dializador de polisulfona en placas que no utilizan PVP como amalgamante, lo cual induce a pensar en un papel significativo de este producto en el desarrollo de la reacción. En ninguno de estos casos el cambio de dializador supuso la desaparición de las crisis y el desarrollo de urticaria crónica con eosinofilia. Otras causas que valorar serían la retrofiltración de endotoxinas en membranas de alta permeabilidad (ausente en membranas de baja permeabilidad), la hipersensibilidad a hierro intravenoso o el uso de inhibidores de la enzima convertidora de angiotensina (IECA).
Estas reacciones han inducido a realizar una evaluación no solo de las ramas y del dializador sino también de los procesos de esterilización que incluyen como principal factor el óxido de etileno y a los diferentes y numerosos medicamentos utilizados en el tratamiento de la Enfermedad Renal Crónica (ERC) como son la heparina, soluciones de hierro, vitaminas y las eritropoyetinas.

Intuir una reacción de hipersensibilidad en este tipo de pacientes es extremadamente difícil por lo que hay que analizar la situación de los pacientes afectados por la enfermedad de base, los estigmas de ERC y las comorbilidades que frecuentemente los afectan.

Las membranas son el elemento fundamental del tratamiento de HD, puesto que a través de ellas se realizan la filtración, la difusión y otros procesos físicos responsables de la detoxificación de la sangre y la eliminación de los metabolitos de desecho orgánico, estas membranas semipermeables tienen su origen en materiales naturales como la celulosa, el papel almidonado y otros derivados del algodón que se usaron en la etapa experimental e inicial de la nefrología en el mundo contemporáneo, precisamente este tipo de membranas eran poco toleradas, lo que obligo a la búsqueda de nuevos materiales que ofrecieran mejores índices de eficiencia y eficacia en la depuración de toxinas y filtración de agua, comenzaron a utilizarse materiales con cualidades físico-químicas donde la

\footnotetext{
${ }^{1}$ Clinica Reynadial, Guayaquil, Ecuador. Hospital General IESS Babahoyo, Los Ríos, Ecuador.

${ }^{2}$ Universidad Técnica de Babahoyo, Ecuador.

${ }^{3}$ Clinica Reynadial. Hospital General IESS Sur Valdivia, Guayaquil, Ecuador.

${ }^{4}$ Clinica Reynadial, Guayaquil, Ecuador.

5Universidad de las Américas, Quito, Ecuador.

Corresponding author: martha.fors@udlanet.ec
} 
porosidad, el espesor, la hidrofilia, la conductividad alcanzaron una diversidad, en la misma medida que se lograba mejorar ostensiblemente la biocompatibilidad.

Las polisulfonas ocupan un lugar importante en el desarrollo de las membranas dializadoras y han generado una indudable mejoría en los resultados del tratamiento hemodialítico, pero también hay evidencias científicas de reportes de hipersensibilidad a este tipo de membranas sintéticas.

La Clínica Reynadial es un centro de atención dedicado a brindar Servicio de Hemodiálisis a pacientes portadores de Enfermedad Renal Crónica grado V. Brinda servicios desde el año 2016 y ha sido fiel seguidora de los Protocolos de tratamiento elaborados por las Autoridades del Ministerio de Salud Pública (MSP) y el Instituto Ecuatoriano de Seguridad Social (IESS), así como una continua actualización de técnicas y procederes tomando como modelo las normas Europeas y Americanas y la medicina basada en evidencia, lo que permite brindar una atención de excelencia en el tratamiento de 140 pacientes.

En términos generales la Hemodiálisis se practica 3 veces por semana durante 4 horas; En REYNADIAL como protocolo se maneja la diálisis Hipotérmica (Temperatura del líquido de dializado en $35^{\circ} \mathrm{C}$ ), con NA ajustado al perfil de los pacientes y sumando monitoreo de la tensión arterial y frecuencia cardiaca, el peso se estima por clínica y ajustes orientados a través de la realización de la medición ultrasónica del diámetro de la vena cava inferior y la Bioimpedancia, de manera tal que los parámetros fundamentales se manejan con estándares que permiten la calidad en hemodiálisis.

En este estudio reportamos un primer caso de hipersensibilidad a membranas sintéticas de polisulfona, en el que se realizó una revisión exhaustiva de todas las potenciales causas de aparición de esta condición como las anafilactoides, hemodinámicas y cardiovasculares. que pudieran explicar la sintomatología presentada que finalmente fueron corregidas al sustituir las membranas de polisulfona por membranas semisintéticas de triacetato, la cual logra un Kt y un volumen convectivo adecuados, con buen perfil de biocompatibilidad e inflamatorio, lo que la convierte en una posibilidad más de tratamiento en pacientes alérgicos a membranas sintéticas.

\section{Reporte de caso}

Paciente NCLQ de 75 años de edad, con antecedentes de Diabetes Mellitus, Tuberculosis pulmonar e Hipertensión Arterial de más de 15 años de evolución, así como Enfermedad Renal Crónica (ERC), que fue referido a Hemodiálisis(HD) el 24 de junio del 2019, con catéter yugular derecho, anemia (hemoglobina $7,7 \mathrm{~g} / \mathrm{l}$ ), hipoalbuminemia discreta, y presentando al examen físico: edemas de miembros inferiores, palidez cutáneo mucosa, con peso de 60.9 kg y tensión arterial (TA) de 160/60.

En el transcurso de los 2 meses iniciales en HD este paciente solo presentó elevación de la tensión arterial durante las sesiones de hemodiálisis, así como una positiva evolución de su síndrome de ERC con desaparición de los edemas en miembros inferiores, ascenso de los valores de Hemoglobina y serinemia, destacando la permanencia de valores absolutos elevados de eosinofilia.

En el mes de octubre (15/10/2019) se realiza exitosamente una fistula arteriovenosa (FAV); desde el punto de vista de la analítica mantenía la eosinofilia, habiendo ya corregido la anemia con hemoglobina en valores de $11 \mathrm{~g} / \mathrm{L}$.

En el mes de noviembre del 2019 el paciente comienza con manifestaciones de diarreas liquidas, sin flemas, ni conte- nido hemático, ni alimenticio, acompañadas de dolor abdominal difuso, sin pujos ni tenesmo al comienzo de la sesión (10 minutos) así como astenia, mejorando pasada una hora en tratamiento (si se mantenía conectado), a veces con necesidad de desconexión y reconexión. En el mes de diciembre, a lo anterior se agregan náuseas, epigastralgia e hipotensión arterial, después de 15 a 20 minutos de conectado a la hemodiálisis, por lo que se investigan otras causas probables especialmente hemodinámicas, ya que se asociaba su morbilidad intradialítica a la previa realización de la FAV ; es de señalar la repetición de estos episodios en cada sesión de hemodiálisis, hasta entonces realizadas con filtros de polisulfona de al menos 3 compañías manufactureras distintas.

Los cambios de filtro a otra polisulfona, definitivamente no se mostraron eficaces en la reducción de los síntomas de este paciente. (Tabla 1)

\begin{tabular}{|c|c|}
\hline Tipo de membrana & FECHA \\
\hline FX 8 & $24 / 6 / 2019$ \\
\hline REVACLEAR 300 & $\mathbf{2 8 / 6 / 2 0 1 9}$ \\
\hline FX 8 & $19 / 8 / 2019$ \\
\hline P18 & $\mathbf{2 / 9 / 2 0 1 9}$ \\
\hline FX 8 & $18 / 9 / 2019$ \\
\hline FX 60 & $\mathbf{1 / 1 1 / 2 0 1 9}$ \\
\hline REVACLEAR 300 & $24 / 12 / 2019$ \\
\hline P 18 & $\mathbf{2 6 / 1 2 / 2 0 1 9}$ \\
\hline SUREFLUX & $28 / 12 / 2019$ \\
\hline
\end{tabular}

Tabla 1. Cambios de membranas al paciente.

Tampoco el uso de hidrocortisona, antihistamínicos y oxígeno con máscara produjeron mejorías ostensibles. Se realiza un ecocardiograma pensando en la posibilidad de un bajo gasto, obteniéndose un resultado normal con F/E 65\%; el electrocardiograma también fue normal; la exploración con ECO de los diámetros de vena cava inferior e índice caval expresaron adecuada volemia. Se observan los resultados de los exámenes de laboratorio en la Tabla 2. La eosinofilia se mantuvo todo el periodo incluso después del cambio de membrana.

Analizando otras posibilidades y teniendo en cuenta informaciones sobre casos de reacción de hipersensibilidad a las membranas sintéticas, se decide pasar a membranas celulósicas eligiendo Sureflux N10 de NIPRO (triacetato de celulosa). Una vez utilizada esta membrana desaparecen inmediatamente todos los síntomas, y manteniéndose asintomático a partir de entonces hasta la fecha de este reporte.

\section{Discusión}

La composición de las membranas dializadoras desde el uso de celulosa a membranas sintéticas, los métodos de esterilización para la eliminación de óxido de etileno y la utilización de otros materiales en el dializador y los tubos como son diferentes pegamentos, aglutinantes, plásticos, látex y siliconas han evolucionado para mejorar la biocompatibilidad. A pesar de esta biocompatibilidad mejorada, no se ha observado una disminución en las reacciones de hipersensibilidad que aparecen en los pacientes que reciben hemodiálisis ${ }^{5,6}$.

Un aumento en el número de informes sobre reacciones 


\begin{tabular}{|c|c|c|c|c|c|c|c|c|c|}
\hline & JUNIO & JULIO & AGOSTO & SEPTIEMBRE & OCTUBRE & NOVIEMBRE & DICIEMBRE & ENERO & FEBRERO \\
\hline & & & & & & & & & \\
\hline LEUCOCITOS & 8.56 & 8.26 & 10.9 & 12.4 & 8.53 & 8.03 & 8.59 & 13.00 & 8.000 \\
\hline EOSINOFILOS \% & 12.5 & 15.3 & 23 & 19.4 & 22 & 29 & 24 & 20.4 & 40.2 \\
\hline CONTEO ABSOLUTO & 1.070 & 1.270 & 2.520 & 2.410 & 1.900 & 0.700 & 0.900 & 2.654 & 1.415 \\
\hline PLAQUETAS & 286.000 & 364.000 & 325.000 & 233.000 & 232.000 & 166.000 & 178.000 & 305.000 & 230.000 \\
\hline
\end{tabular}

Tabla 2. Resultados de exámenes de laboratorio.

de hipersensibilidad ha creado alarma y ha provocado una advertencia por parte de las autoridades sanitarias españolas sobre los riesgos de las membranas de polisulfona ${ }^{1,7,8}$.

Existen reportes de que 1 de cada 20 a 50 pacientes tiene probabilidades de experimentar una reacción anafilactoide con un nuevo dializador, lo que indica que la magnitud del problema es grande ${ }^{9}$. Desde ya hace varios años, se reportó que la incidencia de reacciones era de 0.17 por 1000 sesiones con membranas de celulosa y 4.2 por 1000 sesiones con membranas sintéticas ${ }^{10}$.

La morbilidad intradialítica extrema de este cas nos insinuaba la necesidad de profundizar en la pesquisa de situaciones parecidas pero quizás de menor intensidad que pudieran estar presentes en nuestros pacientes polisintomáticos y relacionadas con membranas sintéticas con la misma probable fisiopatogenia, por lo que decidimos indagar en manifestaciones clínicas de hipersensibilidad (prurito, urticaria, broncoespasmo, opresión precordial, hipotensión arterial, dolor abdominal, vómitos, diarreas, molestias) generadas durante el proceso dialítico proceder ante la duda a cambiar el dializador por celulósico, evaluando resultados clínicos demostrables.

Las manifestaciones en este paciente fueron recidivantes y generadas en el proceso de la hemodiálisis o relacionadas con el uso de una membrana de polisulfona. Al realizar el cambio de ésta, las manifestaciones clínicas desaparecieron.

Puede haber reacciones tanto con celulosa como con membranas sintéticas, aunque las membranas sintéticas, en la actualidad, causan más reacciones alérgicas. Es posible que el uso de otros materiales como la PVP pueda aumentar la probabilidad de sufrir una reacción de hipersensibilidad en estos pacientes.

Se deben descartar otras causas de reacciones alérgicas como látex, hierro intravenoso, heparina y formaldehído en pacientes que sufren reacciones de hipersensibilidad en diálisis.

\section{Conclusiones}

Las reacciones de hipersensibilidad pueden ocurrir con cualquier tipo de membranas, pero el reciente aumento en los casos publicados relacionados con las membranas sintéticas de polisulfona nos advierte de esta posibilidad, por lo que el personal que atiende a pacientes en hemodiálisis debe estar atento a cualquier manifestación de este tipo. El triacetato de celulosa parece ser una buena alternativa para estos pacientes.

\section{Referencias bibliográficas}

1. Sánchez-Villanueva Rafael J., González Elena, Quirce Santiago, Díaz Raquel, Álvarez Laura, Menéndez David et al. Reacciones de hipersensibilidad a membranas sintéticas de hemodiálisis. Nefrología (Madr.) [Internet]. 2014 [citado 2020 Jul 01]; 34(4): 520-525. Disponible en: http://scielo.isciii.es/scielo.php?script=sci_arttext\&pid=S0211-69952014000400012\&lng=es. http:// dx.doi.org/10.3265/Nefrologia.pre2014.May.12552.

2. Arenas MD, Gil, MT, Carretón MA, Moledous A, Albiach B. Efectos adversos a polisulfona durante la sesión de hemodiálisis. Nefrología, 27(5):638-642

3. Caruana RJ, Hamilton RW, Pearson FC. Dialyzer hypersensitivity syndrome: possible role of allergy to ethylene oxide. Report of 4 cases and review of the literature. Am J Nephrol 1985;5:271-4.

4. Kuragano T, Kuno T, Takahashi Y, Yamamoto C, Nagura Y, Takahashi S, et al. Comparison of the effects of cellulose triacetate and polysulfone membrane on GPIllb/lla and platelet activation. Blood Purif 2003;21:176-82

5. Ebo DG, Bosman JL, Couttenye MM, Stevens WJ. Haemodialysis-associated anaphylactic and anaphylactoid reactions. Allergy 2006;61:211-20.

6. Butani L, Calogiuri G: Hypersensitivity reactions in patients receiving hemodialysis. Ann Allergy Asthma Immunol 2017; 118: 680-684.

7. González Sanchidrián S, Labrador Gómez PJ, Marín Álvarez JP, Jiménez Herrero MC, Castellano Cerviño I, Gallego Domínguez S, Sánchez-Montalbán JM, Deira Lorenzo J, Davin Carrero E, Planco Candelario S, Gómez-Martino Arroyo JR: Reacción a membranas sintéticas en hemodiálisis. Nefrología 2016; 36: 707-709.

8. Martín-Navarro JA, Gutiérrez-Sánchez MJ, Petkov-Stoyanov V: Hypersensitivity to synthetic hemodialysis membranes. Nefrologia 2014; 34: 807-808.

9. Nicholls AJ. Hypersensitivity to hemodialysis: the United Kingdom experience. Artif Organs. 1987;11(2):87-89. doi:10.1111/j.1525-1594.1987.tb02634.x

10. Simon P, Potier J, Thebaud HE. Facteurs de risque des réactions aiguës d'hypersensibilité en hémodialyse: enquête prospective multicentrique sur six mois dans l'Ouest de la France [Risk factors for acute hypersensitivity reactions in hemodialysis]. Nephrologie. 1996;17(3):163-170

Received: 24 septiembre 2020

Accepted: 10 octubre 2020 